\title{
Personal Chart: Health Data Logging Made Easy with a Mobile Device
}

\author{
Mikko Paldanius ${ }^{1}$, Anu Lehtiö ${ }^{2}$, \\ Minna Karukka ${ }^{1}$, and Pertti Huuskonen ${ }^{2}$ \\ ${ }^{1}$ Nokia Research Center, Yrttipellontie 10, 90230 Oulu, Finland \\ ${ }^{2}$ Nokia Research Center, Visiokatu 1, 33720 Tampere, Finland \\ firstname.surname@nokia.com
}

\begin{abstract}
Many devices are still unconnected. We apply mobile imaging to log measurements from personal health devices. Such devices sometimes offer wired and wireless links, but they suffer from many problems (setup, breaking connections, non-mobility). We propose to use instead ubiquitous mobile phone cameras to capture the measurements and store them for further viewing and follow-up. In this paper we discuss the principle, the prototype, the user study and initial conclusions of this approach.
\end{abstract}

Keywords: Healthcare, human factors, optical character recognition.

\section{Introduction}

Today's personal health data measurement devices (e.g. blood pressure meters, glucose monitors) are still to a large degree disconnected. Their users need to keep track of the measurements manually, and transfer the measurements to logbooks or analysis software. This obviously is tiresome and creates a bottleneck for automated health monitoring. The same issues have existed a long time in clinical environments. With decreasing staff and increasing amounts of data, medical workers would appreciate automated data logging from their instruments.

Many clinical and some consumer devices do feature wired or wireless links for transferring the data to PCs and eventually to internet services. There are also devices that connect to mobile phones for the same functionality [1, 2]. However, in too many cases the users need to take elaborate steps to initiate the data transfer. Wireless technology is still difficult for people who are averse to technology: convoluted setup procedures and intermittent connections confuse them. The problem is worse for mobile use situations, where available interaction times may be reduced to seconds [3]. In earlier research e.g. gestures have been suggested for more intuitive way of pairing devices and interacting with other devices $[4,5]$.

We propose a different approach. Instead of relying to wireless links or cables, we use mobile imaging for data transfer. A large fraction of mobile phones in the market already include suitable hardware for the purpose. The phone camera can take a photo of the display of a personal measurement device, interpret the measurement reading through OCR (Optical Character Recognition), and store the data for later retrieval or uploading into a web service. This gives people a fast, convenient way to keep track 
of their health data. It avoids problems related to wireless links, empowering people to use an interaction method they already know: point and shoot. In this paper we introduce and evaluate an implementation of this approach on mobile phones.

\section{Personal Chart Application}

Personal Chart for Nokia N900 is an application for detecting blood pressure values from a measuring device. Personal Chart has two main functionalities: capturing blood pressure values and viewing measurement history. In addition, users can share their personal measurement information via Bluetooth or email. After the application is launched for the first time, a user is asked to create a profile. Profile is made up of name, gender and weight. The application enables using several profiles.

Capturing is launched by clicking the camera icon on the start view. A user is guided to point camera to measuring device and match the live view screen marks with the display of measuring device. Values can be entered manually by pressing skip tracking icon. Once the values are detected from the live view, the confirmation view is opened. The user can manually alter the values in case of misdetection. In the confirming view the user can save values or backtrack to capturing mode.

Measurement history can be viewed as a table layout and a graphic line chart. In the table view, a user can see all the values in chronological order. In the chart view, presented in figure 1, measurement history is portrayed in a line graph according to measured value and time. The user can choose which lines are drawn in the graph by toggling the check boxes. Saved data can be shared via email or Bluetooth. Shared file is a CSV file (Comma Separated Values) that opens in almost all spreadsheets, including Microsoft Excel and OpenOffice Calc.

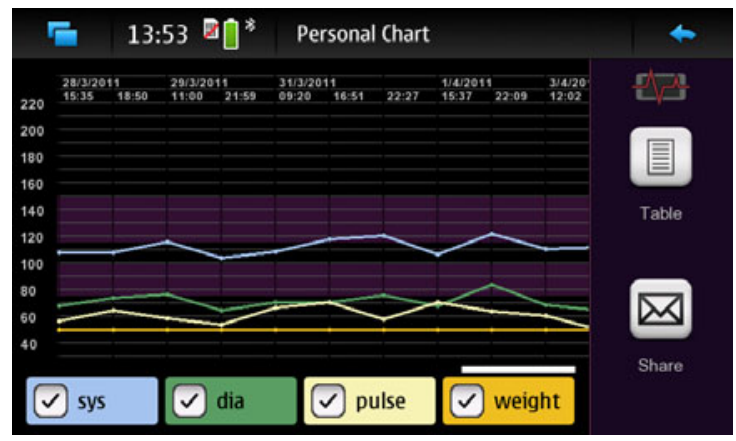

Fig. 1. Chart view of the Personal Chart application

\section{Evaluation}

The main goal of our study was to evaluate the general acceptability of the application concept and the applied technology. Basic requirements for participants were chosen to be the age of 25-55 years, experience in smart phones and the use of touch screen 
devices. It was preferred that both sexes are represented. Evaluation was based on user testing session and voluntary online discussion. Originally the interviews and the discussions were in Finnish.

\subsection{User Testing}

The total amount of 50 users was considered to be sufficient to give reliable and valid results. Each participant took part in 30 minute user testing session, 31 of them were male and 19 female. During the test the users were given 3 tasks, after performing each task they answered open-ended questions and graded statements related to that. After the session, users answered open-ended questions concerning the application as a whole and filled in the UX -questionnaire.

The first flow of tasks were to create a user profile, check how the used device is selected, to measure blood pressure, to transfer the readings to mobile device first by tracking then manually and save them. Creating a profile was considered easy and the add user button was clear and well placed. In addition to name, gender and weight, suggested adds for profile were age and body mass index. After creating a profile, users moved on to device selection. Choosing the device model and manufacturer was considered easy, but the name of the menu was causing confusion. OCR was the preferred method to manual inputting: Easiness, quickness, modernity and avoiding the extra step of typing were typical reasons beyond that. Manual inputting could be improved by making it more intuitive. Those few who preferred manual input had the most trouble with the lightning conditions, optical character recognition is remarkably vulnerable due to the application`s sensitivity to shadows and reflections. Saving the data was easy and not a single user had trouble with that.

The second task was to view the blood pressure data in table and chart form and to administer the graphs shown in the chart view. The chart view was preferred to the table view because of giving better overall view of development. In addition, it was argued to be visually more appealing, more illustrative and the graphs were easily distinguishable and fun. The shifting between the different views was easy and fast. In the chart view, many users were asking for some feedback from their blood pressure readings. Despite of the preference towards the chart, both views were needed. They serve a different purpose: the chart is good for getting an overall view on trends and the table gives you exact values and detailed information.

The third task was to share own blood pressure data. Users preferred Email to Bluetooth. It was considered easy and more familiar. Most likely recipients were doctor, nurse, coach and personal trainer. In addition, users wanted to send the data to themselves with the intention to storage and view the data with another device. Especially these users were interested in the possibility to use Bluetooth. The possibility to send the data forward was seen useful and add value to the use of the application. In the case of annual health examination, the data could be sent to the doctor beforehand to make analyzing and medication planning more effective. In addition, individuals could decrease the amount of visiting the doctor by measuring blood pressure unassisted and sending the data by themselves, also from abroad. 


\subsection{Online Discussion}

After the user testing, users had an option to air one`s opinion in the discussion forum for three weeks. 11 male and 5 female took part in discussion with 5 different topics given by moderator. The best thing was argued to be the simplicity and the ability to share information. Participants defined quite unanimously that the most suitable users for this application would be the people who have to follow their blood pressure values and nursing staff who can send the values forward. Improvement and developing ideas consisted of alternative methods for data transfer, a PC client for the application and some changes for the user interface.

\section{Conclusions}

The Personal Chart application was considered modern and easy to use for health data logging. However, we believe that UI enhancements and more flexible character recognition would have a clear positive effect on usability of this application. In the health and well-being research, Personal Chart could create a valuable channel for collecting large amount of collaborative health data. Before that, user evaluations with doctors and nurses could be a fertile ground for future investigations.

In general, Personal Chart was seen useful and participants found many use cases for the application related to personal health management and communication with health care. It could bring true value for both doctors and patients by making their interaction with each other more effective and easier. In addition, blood pressure and blood sugar data could be easily updated from mobile phone into popular services related to exercise, nutrition and weight-watching. Since people are nowadays encouraged to take good care of themselves, an application that supports user's knowledge of his/her own health seems to be very current. In addition, this technology could suit a whole variety of applications for non-medical devices, for example with industrial maintenance.

\section{References}

1. Polar WearLink,

http: //www.polar.fi/en/products/accessories/WearLink_transmit ter

2. Sports Tracker, http: //www. sports-tracker.com/

3. Oulasvirta, A., Tamminen, S., Roto, V., Kuorelahti, J.: Interaction in 4-second bursts: the fragmented nature of attentional resources in mobile HCI. In: Proceedings of the SIGCHI Conference on Human Factors in Computing Systems, pp. 919-928. ACM, New York (2005)

4. Kray, C., Nesbitt, D., Dawson, J., Rohs, M.: User-defined gestures for connecting mobile phones, public displays, and tabletops. In: Proceedings of the 12th International Conference on Human Computer Interaction with Mobile Devices and Services, pp. 239-248. ACM, New York (2010)

5. Peng, C., Shen, G., Zhang, Y., Lu, S.: Point\&Connect: intention-based device pairing for mobile phone users. In: Proceedings of the 7th International Conference on Mobile Systems, Applications, and Services, pp. 137-150. ACM, New York (2009) 